\title{
ANÁLISE DA COMPOSIÇÃO QUÍMICA DO ÓLEO ESSENCIAL DE CAPIM SANTO (CYMBOPOGON CITRATUS) OBTIDO ATRAVÉS DE EXTRATOR POR ARRASTE COM VAPOR D’ÁGUA CONSTRUÍDO COM MATERIAS DE FÁCIL AQUISIÇÃO E BAIXO CUSTO
}

\author{
F. F. M. SILVA ${ }^{1}$, L. F. MOURA, P. T. BARBOSA, A. B. D. FERNANDES, L. M. BERTINI ${ }^{2}$ e L. A. ALVES \\ Instituto Federal de Educação, Ciência e Tecnologia do Rio Grande do Norte - Campus Apodi. \\ felipe.maia@ifrn.edu.br ${ }^{1}$, luciana_bertini@hotmail.com²
}

Artigo submetido em novembro/2013 e aceito em dezembro/2013

DOI: 10.15628/holos.2014.1762

\section{RESUMO}

Os óleos essenciais são substâncias naturais de grande importância econômica, sendo utilizados em perfumaria, cosméticos, alimentos e medicamentos. São obtidos por diferentes métodos de extração e encontrados em diferentes partes de plantas aromáticas. Contudo, a aquisição de equipamentos para extração de óleos essenciais representa um custo significativo para as instituições de ensino e pesquisa do país, principalmente nos últimos anos em que tem ocorrido redução de investimentos nas áreas de Ciência e Tecnologia. Desta forma, esse trabalho teve como objetivo a construção de um extrator de óleo essencial utilizando materiais de fácil acesso e mão de obra local. 0 extrator construído possui as dimensões de $60 \mathrm{~cm}$ de altura e $30 \mathrm{~cm}$ de diâmetro. Seu aquecimento é realizado por resistência elétrica com temperatura controlada por termostato (faixa de 0 a $120^{\circ} \mathrm{C}$ ) e possui uma campânula metálica vedada por selo d'água que está acoplada a um sistema de condensação. $\mathrm{O}$ experimento teste foi realizado fazendo extração de $612 \mathrm{~g}$ de capim santo (Cymbopogon citratus). Após análise obteve-se um rendimento de $0,48 \%$ de óleo essencial na extração. Portanto, este trabalho demonstra a busca no desenvolvimento de tecnologias inovadoras, fazendo uso de materiais facilmente encontrados no mercado e utilizando mão de obra local, contribuindo, não só para o acúmulo de conhecimento acadêmico, mas, acima de tudo, com a capacitação de recursos humanos, geração de renda local e com a transferência de novas tecnologias que possam servir de base para a elaboração de novos produtos, neste caso, os óleos essenciais.

PALAVRAS-CHAVE: Óleo essencial, extrator, capim santo, tecnologias inovadoras.

\section{ANALYSIS OF CHEMICAL COMPOSITION OF THE ESSENTIAL OIL OF HOLY GRASS (CYMBOPOGON CITRATUS) OBTAINED THROUGH EXTRACTOR FOR WATER VAPOR TRAIL WITH BUILT WITH MATERIALS OF ACQUISITION AND EASY LOW COST}

\begin{abstract}
Essential oils are natural substances of great economic importance, being used in perfumery, cosmetics, food and medicines. They are obtained by different extraction methods and found in different parts of plants. However the purchase of equipment for the extraction of essential oils has a significant cost to the education and research institutions in the country, especially in recent years that there has been a reduction of investment in the areas of Science and Technology. Thus, this study aimed to construct an essential oil extractor using accessible materials and local labor. The extractor constructed having the dimensions of $60 \mathrm{~cm}$ height and $30 \mathrm{~cm}$ in diameter. Its heat is carried out by temperature
\end{abstract}

controlled electrical resistance thermostat (range 0 to $120^{\circ} \mathrm{C}$ ) and has a metal hood sealed by seal water that is coupled to a condensing system. The experimental test was accomplished by extracting $612 \mathrm{~g}$ of holy grass (Cymbopogon citratus) giving a yield of $0.48 \%$ essential oil extraction. Therefore, this work demonstrates the possibility of developing innovative technologies, using materials easily found in the market and using local labor, contributing not only to the accumulation of academic knowledge, but above all, with the training of human resources, local income generation and transfer of new technologies that can serve as a basis for the development of new products, in this case, the essential oils.

KEYWORDS: Essential oil extractor, lemongrass, innovative technologies. 


\section{INTRODUÇÃO}

Os óleos essenciais compreendem uma mistura de substâncias voláteis extraídas de plantas aromáticas e são encontrados nas partes aéreas (folhas e ramos finos), cascas, troncos, raízes, frutos, flores, sementes e resinas (ZOGHBI, 1998). Segundo Santos (2004) e Bajpai (2008) esses óleos são formados por diversas classes de ésteres de ácidos graxos, mono e sesquiterpenos, fenilpropanóides, alcoóis, aldeídos, e em alguns casos, por hidrocarbonetos alifáticos, entre outros. Em seu trabalho, Viana (1998) relata a grande importância de estudos envolvendo a composição química volátil de plantas, sendo que a classe mais comum é a dos terpenos.

Com o avanço da tecnologia e a globalização, nos últimos anos o homem tem buscado novas descobertas na área medicinal e de cosméticos. Tais avanços despendem grande esforço na obtenção de novas substâncias químicas para o uso farmacológico, através de estudos científicos realizados na fauna e flora brasileira. Os óleos essenciais constituem uma matéria-prima de grande importância na área industrial, utilizados na produção de cosméticos, na indústria farmacêutica e alimentícia, sendo geralmente os componentes de ação terapêutica das plantas medicinais.

Dentre as diversas propriedades biológicas relatadas nos estudos envolvendo óleos essenciais destacam-se os efeitos alelopáticos, antimicrobianos, citotóxicos, hipoglicemiantes e anti-inflamatórios (LOAYZA, 1993; 1995; DUARTE, 2004; BARBOSA-FILHO, 2005; FERRONATT, 2007). Estes trabalhos evidenciam a importância do aprofundamento dos estudos e técnicas de extração dessas classes de compostos tão ricas, tanto quanto a diversidade estrutural de componentes químicos, como pelos comportamentos fisiológicos diversos demonstrados.

Apesar do número relevante de publicações envolvendo estudo sobre a composição química, atividade biológica e derivatização dos óleos essenciais, quando se leva em consideração a riqueza da flora brasileira, percebe-se que ainda há muito que se explorar dentro de uma das maiores e mais diversificadas fontes do mundo.

Giulietti (2005) afirma que mesmo com o número de investigações realizadas até o momento a respeito da flora brasileira o estudo sobre as espécies vegetais no Brasil ainda é um campo muito novo visto a enorme diversidade de espécies vegetais existentes e ao pequeno número de espécies investigadas. Só no Brasil, o número de espécies de plantas ultrapassa os 56000, o que compreende quase $19 \%$ da flora mundial, sendo que o conhecimento da biodiversidade no país ainda é muito incompleto.

A obtenção destes compostos voláteis pode ser realizada por diversos procedimentos, tais como: hidrodestilação, arraste com vapor d'água, extração a frio e a quente com solvente, enfleuragem, $\mathrm{CO}_{2}$ supercrítico e outros (CASSEL, 2006; SCHANEBERG, 2002). Entretanto, os métodos de hidrodestilação e arraste com vapor de água são os mais utilizados. Apesar de apresentarem semelhanças entre si, à composição química do óleo obtido por estes processos podem ser acentuadamente diferentes, uma vez que a hidrodestilação constitui-se em um método em que o material vegetal fica em contato direto com a água em ebulição, onde os constituintes voláteis são arrastados pelo vapor de água e em seguida, condensados em sistema de refrigeração. Já o processo de arraste com vapor d'água tem a parte vegetal da qual se deseja obter o óleo 
separado da água em ebulição, esta gera o vapor que é direcionado para o material vegetal, arrastando os compostos voláteis que serão em seguida condensados (SIMÕES et al., 1999).

Devido à riqueza de constituintes químicos presentes nos óleos essenciais, estes continuam a atrair cada vez mais estudos visando ampliar suas aplicações e potencializar suas propriedades. Contudo, a aquisição de equipamentos utilizados na extração destes óleos representa um custo significativo para muitos pesquisadores. Pensando nisso, este trabalho teve como objetivo construir um sistema de extração de óleos essenciais por arraste com vapor d'agua, com o apoio de profissionais locais (Cidade de Apodi/RN), usando materiais de fácil aquisição no comércio e ainda extrair o óleo de capim santo comparando a sua composição química e rendimento com os já relatados na literatura.

\section{MATERIAIS E MÉTODOS}

\subsection{Fabricação do Extrator}

Os materiais utilizados para construção do extrator foram: chapa de ferro de $(2 \mathrm{mx} 1 \mathrm{~m})$ com $1,2 \mathrm{~mm}$ de espessura, uma válvula de contenção de $25 \mathrm{~mm}$, dois spray $(150 \mathrm{~g})$ de tinta de alta temperatura e solda oxiacetilênica. Todos os materiais foram adquiridos no comércio local da cidade de Apodi-RN. A resistência elétrica e o termostato de temperatura regulável foram comprados em lojas especializadas no município de Fortaleza-CE.

A chapa de ferro foi modelada em formato cilíndrico e suas partes soldadas com solda oxiacetilênica e adaptado a resistência elétrica com o termostato para o sistema de aquecimento de água juntamente com uma válvula de drenagem do extrator. No seu interior foi colocada uma peneira metálica para a separação da matéria vegetal e da água em ebulição. Por fim, foi confeccionada uma campânula metálica com vedação através do selo d'agua existente na parte superior do extrator (Figura 01).

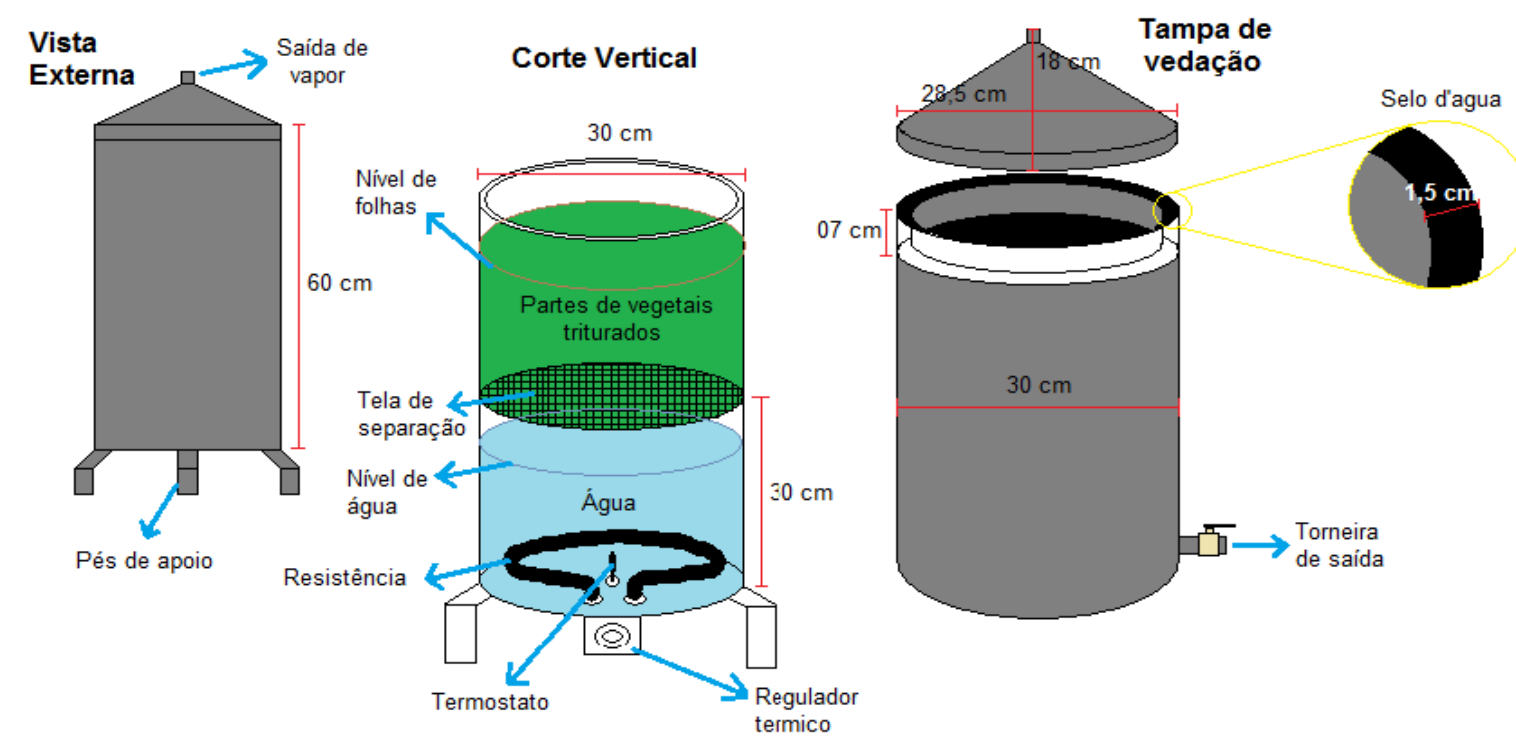

Figura 01: Dimensões do Extrator do Óleo essencial. Fonte: Maia Silva (2013) 


\subsection{Método de Extração}

Para a extração do óleo essencial foi utilizado 612 gramas de capim santo (Cymbopogon citratus), coletadas no Instituto Federal de Educação, Ciência e Tecnologia do Rio Grande do Norte, Campus Apodi. O material vegetal fresco foi cortado em pequenas partes e inserido dentro do extrator que já continha $10 \mathrm{~L}$ de água no seu interior. Em seguida, a campânula metálica foi selada com água e o equipamento foi acoplado a um sistema de resfriamento como pode ser observado na Figura 02.

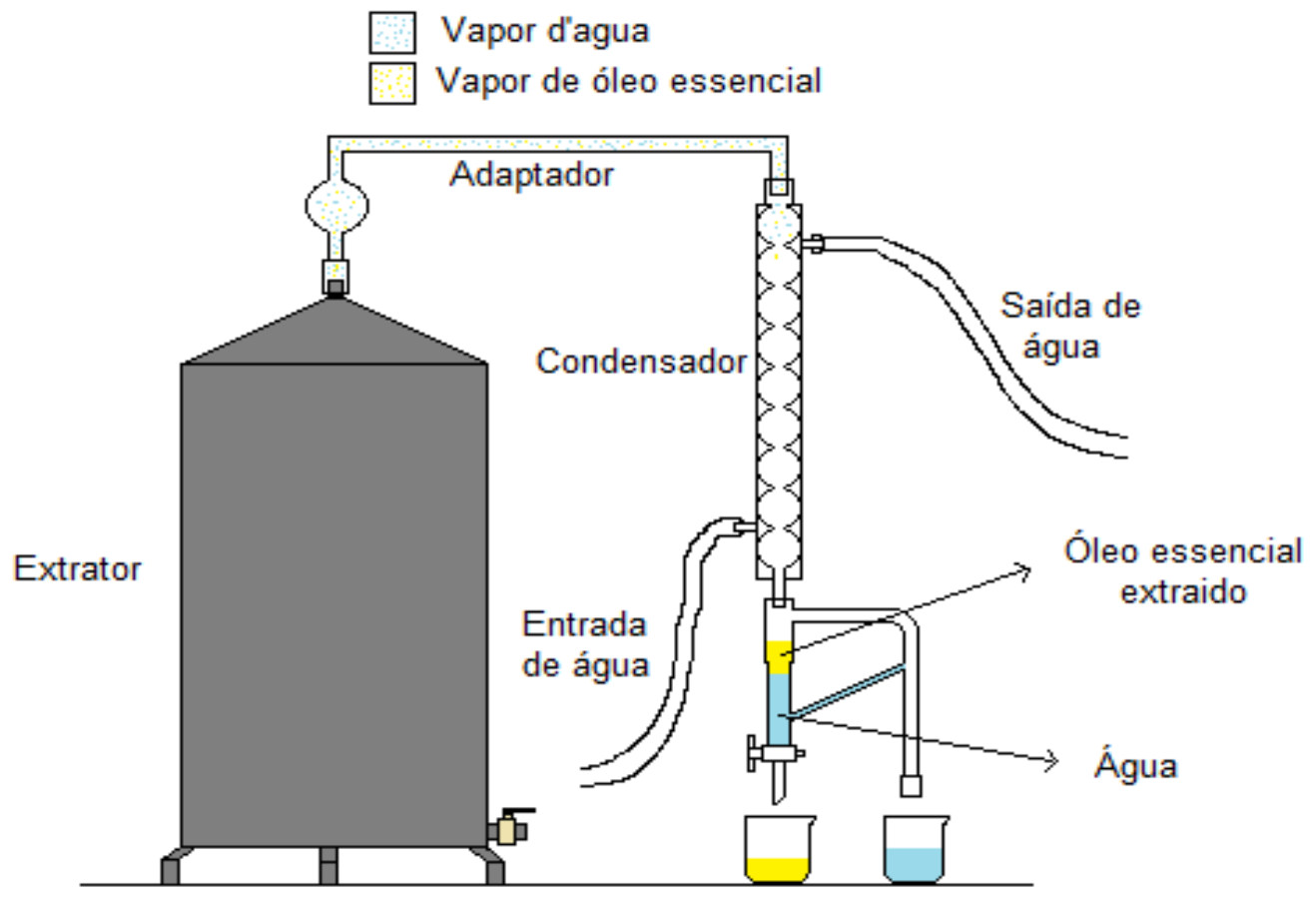

Figura 02: Sistema de Extração do Óleo essencial. Fonte: Maia Silva (2013)

Após o sistema montado e já com a matéria vegetal em seu interior, a resistência foi ligada para efetuar o aquecimento da água em seu interior. A extração foi efetuada em triplicata e o tempo de extração (desde o início do aquecimento) foi de 5 horas ininterruptas.

\subsection{Determinação da Composição química dos óleos essências}

A caracterização química dos óleos essenciais foi obtida através de análise em Cromatógrafo Gasoso acoplado a espectrômetro de massa (CG-EM) de marca CGMS-QP2010-SESHIMADZU com tempreratura do injetor a $250^{\circ} \mathrm{C}$, Interface a $300^{\circ} \mathrm{C}$, fonte de íon a $260^{\circ} \mathrm{C}$ com coluna Restek-RTX. 5MS (crossbond ${ }^{\circledR} 5 \%$ difenil/95\% dimetil polisiloxano (crossbond ${ }^{\circledR}$ 5\% difenil/95\% dimetil polisiloxano) 30 metros de comprimento, diâmetro interno de 0,25mm e espessura do filme igual a $0,25 \mu \mathrm{m}$. A temperatura inicial da coluna foi de $40^{\circ} \mathrm{C}$ seguido de aumento a uma taxa de $4^{\circ} \mathrm{C} / \mathrm{min}$ até a temperatura de $180^{\circ} \mathrm{C}$ depois uma elevação de $20^{\circ} \mathrm{C} / \mathrm{min}$ até a temperatura de $280^{\circ} \mathrm{C}$, onde permaneceu por 10 minutos. A quantificação dos componentes foi feita pelo método da normalização das áreas referentes a cada componente obtida por Cromatografia gasosa acoplada a detector de ionização em chama (CG-DIC), marca SHIMADZUM, 
modelo CG 2010 com auto injeto AOC-20i, onde se usou a mesma coluna, o mesmo método e o mesmo gás de arraste utilizados para o CG-EM.

\section{RESULTADOS E DISCUSSÕES}

Após análise do rendimento e da composição do óleo, em comparação com métodos convencionais de extração observou-se que o extrator construído (Figura 03) apresentou desempenho satisfatório quanto ao sistema de vedação, pois, não foram observados vazamentos. O tempo médio para início da condensação do óleo foi de cerca de 40 minutos, sendo possível perceber a formação de gotículas de óleo essencial.

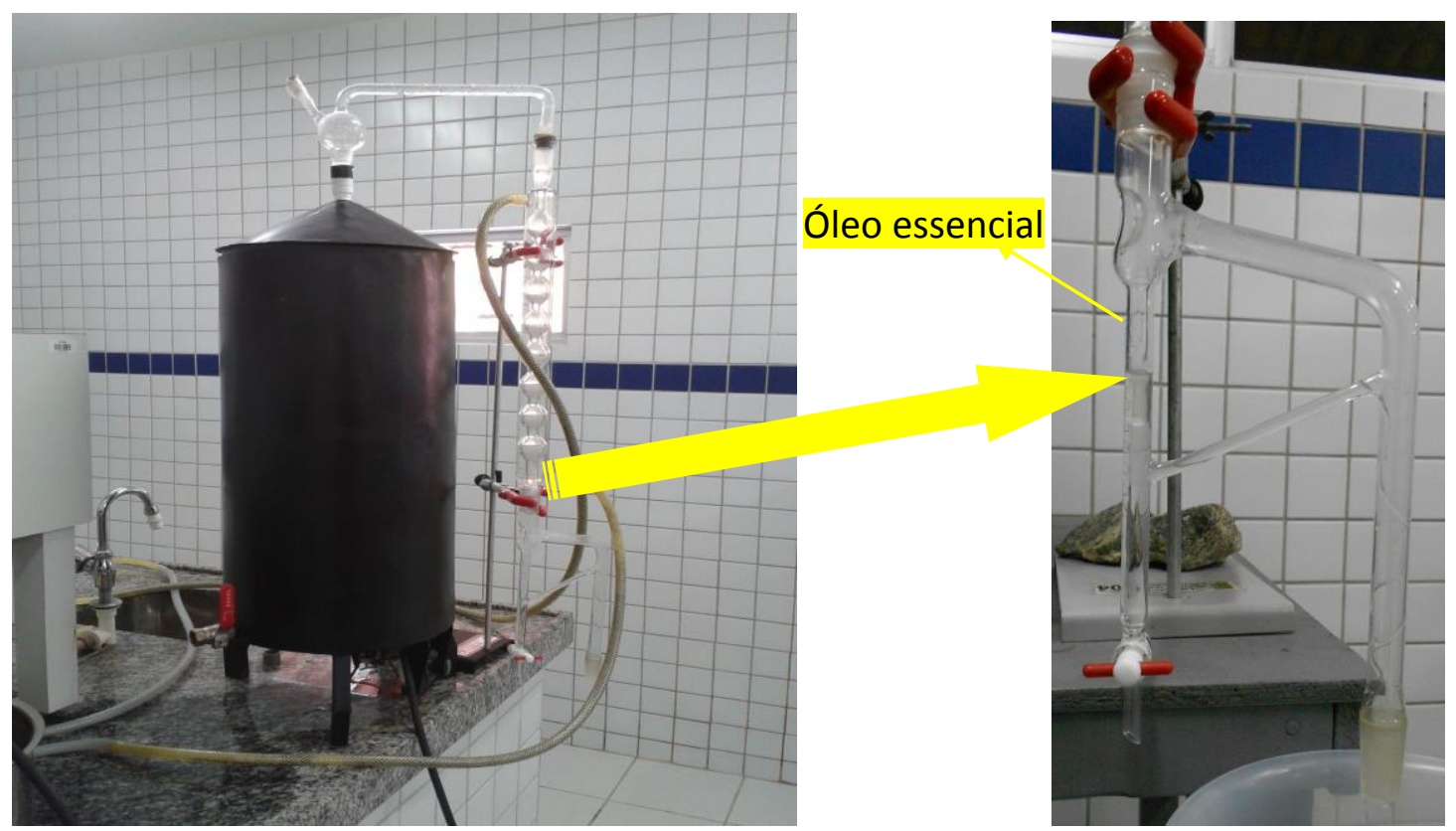

Figura 03: Extrato de óleo construído em funcionamento.

Fonte: Maia Silva (2013)

O rendimento do óleo essencial apresentado pelo extrator foi comparado ao método convencional de hidrodestilação como apresentado na Tabela abaixo.

Tabela 1: Comparação dos rendimentos de extração de óleo essencial

\begin{tabular}{c|c|c}
\hline Método de extração & Rendimento obtido (\%) & Rendimento da literatura (\%) \\
\hline Hidrodestilação & 0,544 & 0,80 (MATASYOH et al, 2011) \\
\hline Arraste com vapor & 0,448 & 0,90 (GBENOU et al, 2012) \\
\hline
\end{tabular}

Quando comparados com dados da literatura (MATASYOH et al, 2011; GBENOU et al, 2012) observou-se que foi obtido um menor rendimento, tanto na hidrodestilação quanto no arraste a vapor, o que não indica relação direta com a construção e eficiência do protótipo de extrator, uma vez que no sistema tradicional de hidrodestilação apresentou uma redução equivalente, ou seja, outros fatores podem estar influenciando na quantidade de óleo essencial extraído, como aponta dados da literatura (SCHWOB, 2004; ANGELOPOULOU, 2002; PALÁ-PAÚL, 2001; CONEJERO, 2001; 
LOPES, 1997; PITAREVIC, 1984) que destacam fatores tais como a sazonalidade, tipo de solo, horário de coleta, idade do vegetal, forma de cultivo, método de extração entre outros.

A composição química do óleo essencial obtido por hidrodestilação e arraste a vapor pode ser analisada na Tabela 2.

Tabela 2. Composição química do óleo 149essencial do Capim santo

\begin{tabular}{|c|c|c|}
\hline Constituinte & Hidrodestilação & Arraste a vapor \\
\hline$\alpha$-citral & 20,63 & 44,69 \\
\hline$\beta$-Citral & 14,50 & 32,42 \\
\hline$\beta$-Mirceno & - & 10,72 \\
\hline Acetato de n-propila & 4,64 & - \\
\hline Acetato de isobutila & 2,29 & - \\
\hline 2,7-Dimetiloct-2,7-dienol & 10,79 & - \\
\hline Tetradecino & 17,14 & - \\
\hline $\begin{array}{ll}\text { Ácido } & \text { 3-(but-3enil)-2,2- } \\
\text { dimetilciclopropanóico } & \end{array}$ & 3,00 & - \\
\hline Verbenol & - & 1,95 \\
\hline Nerol & - & 3,07 \\
\hline Ciclooctanona & 3,02 & - \\
\hline Ácido Nerólico & 1,80 & - \\
\hline
\end{tabular}

Pela a análise dos componentes e suas concentrações obtidas pelos diferentes métodos de extração pode-se perceber que a hidrodestilação apresenta um maior número de substâncias químicas quando comparado com o método de arraste com vapor. Isso pode ser devido ao fato de na hidrodestilação ocorrer o contato direto do material vegetal com a água em ebulição, ou seja, um meio com uma mistura complexa de substâncias submetida à alta temperatura, condições que propiciam varias reações, podendo formar vários artefatos (produtos que não estão presentes no óleo in natura, mas que são formados durante o processo de extração) (SIMÕES, 1999). Quanto à composição, os dois métodos apresentaram como constituintes majoritários o neral e o geranial, resultados que coincidem com os relatados por Oliveira (2011) que obteve estes dois constituintes totalizando $73,83 \%$ da constituição do óleo essencial de Capim santo, usando o método da hidrodestilação. Já a composição apresentada (32,42\% de Neral, 44,69\% de Geranial e 10,72\% de Mirceno, totalizando $87,83 \%$ da composição total) pelo método de extração a vapor se assemelha a relatada por Gbenou (2013), que obteve mirceno $(27,83 \%)$, neral $(19,93 \%)$ e geranial $(27,04 \%)$ totalizando $74,80 \%$ da constituição do óleo essencial de $C$. Citratus, utilizando este mesmo método de extração. Wenqiang (2007) realizou a extração de cravo-da-índia (Eugenia caryophyllata Thunb) por hidrodestilação e arraste a vapor. A constituição majoritária encontrada por ele também foi semelhante em ambos os métodos.

Estes resultados demonstram a possibilidade do uso deste extrator para a obtenção de óleos essenciais, apresentando como vantagem um considerável volume útil para acomodação do material vegetal, o que o torna um diferencial sobre os sistemas de hidrodestilação, que dependendo da densidade do material vegetal, não possibilita a extração de uma quantidade significativa de óleo em uma única batelada. Destaca-se também o fato do extrator ser construído em ferro, o que o torna mais resistente e passível de alterações e modificações, (adaptações, inserção de drenos, sensores, termômetros, manômetros entre outros) características estas, que as vidrarias (balão de fundo redondo, adaptadores), manta aquecedora e outros equipamentos que são utilizadas mais rotineiramente em laboratório para estas atividades não possuem, pois, 
são confeccionadas em vidro. Quanto ao custo também observa-se vantagens na construção do extrator, pois para sua confecção fora gastos 820,00 (Oitocentos e vinte Reais). Se comparados com um sistema convencional de hidrodestilação, com uma manta aquecedora e balão de fundo redondo com capacidade de $5000 \mathrm{ml}$, volume suficiente para suportar a massa testada $( \pm 600 \mathrm{~g})$, o custo gira em torno de até 1500,00 (Um mil e quinhentos reais), dependendo da marca dos equipamentos.

\section{CONCLUSÃO}

Diante dos fatores que dificultam os processos de extração de óleos essenciais pelos métodos tradicionais, tais como: o alto custo dos equipamentos, dificuldade de manutenção e fragilidade das vidrarias, a construção de um extrator utilizando materiais de baixo custo e de fácil aquisição torna-se viável visto que os óleos essenciais tem um abrangente uso na sociedade, como na indústria farmacêutica, cosmética e alimentícia, o que acarreta uma diversidade de benefícios à população consumidora dos produtos oriundos da extração de plantas aromáticas. Tais produtos, por serem considerados naturais, têm apresentado grande demanda e aceitação por parte do mercado consumidor.

Destaca-se outro ponto importante no desenvolvimento deste projeto, que é a incorporação de mão de obra local na construção do equipamento, fato este, que, sem dúvida, em muito contribui para formação técnica e humana da comunidade, ou seja, além de alunos e professores, outros profissionais com talentos muitas vezes não reconhecidos, tornam-se protagonistas do desenvolvimento local, abrindo precedentes para que com estudos futuros, novas culturas sejam comercializadas com um alto valor agregado. Portanto, não se trata de vender o produto primário em si (como é o caso da soja, milho, feijão e outros), mas sim produto de alto valor de mercado, sendo incorporado, ao mesmo, tecnologia e inovação. Estas ações fortalecem toda a cadeia produtiva e apresenta impactos sociais relevantes à medida que tais estudos podem possibilitar um desenvolvimento mais sólido e digno da agricultura familiar, principalmente, em uma cidade onde boa parte de sua população ainda reside e tira seu sustento do meio rural, a Cidade de Apodi-RN.

De maneira sucinta pode-se destacar algumas vantagens na construção do extrator na própria região das Instituições de Ensino, tais como despertar o incentivo pela pesquisa nos alunos, à medida que os mesmos tornam-se os principais indivíduos envolvidos no desenvolvimento da sua formação, ou seja, o aluno não é considerado apenas depósito de um número infinito de informações e sim um agente de transformação. Esta ação apresenta para a comunidade as tecnologias que podem corroborar para com o desenvolvimento local, diversificando e somando valores aos produtos regionais além de contribui de forma relevante para formar os pilares base para o beneficiamento de produtos na região, gerando com isso emprego e renda desde as etapas de produção (plantio e colheita das plantas aromáticas) até a extração e venda dos produtos (no caso, os óleos essenciais). Possibilitando assim a qualificação da mão de obra local, pois, a mesma vai ser orientada para produção de novos produtos e tecnologias, podendo fornecer uma nova opção de renda para os trabalhadores rurais, já que os mesmos podem cultivar espécies aromáticas para produção e extração de óleos essenciais concomitantemente com outras culturas tradicionais. 
Importante ressaltar que novos estudos devem ser realizados para o aprimoramento destas tecnologias assim como outras espécies de plantas aromáticas devem ser testadas visando-se verificar eventuais falhas e possíveis aprimoramentos no sistema, assim como políticas públicas (Incentivo e capacitação dos produtores rurais, apoio a pesquisa) devem ser implantadas para que o sucesso de uma tecnologia inovadora seja alcançado.

\section{AGRADECIMENTOS}

Ao senhor Francisco Everardo de Lima Paiva, profissional local que nos ajudou na confecção do extrator, através de sua experiência e habilidade com solda oxiacetilênica.

À Universidade Federal do Ceará, Departamento de Química Orgânica e Inorgânica, pela análise dos óleos essenciais.

\section{REFERÊNCIAS}

1. ANGELOPOULOU, D.; DEMETZOS, C.; PERDETZOGLOU, D. Diurnal and seasonal variation of the essential oil labdanes and clerodanes from Cistus monspeliensis L. leaves. Biochemical Systematics and Ecology, 30, 189-203, 2002.

2. BAJPAI, V.K.; RAHMAN, A.; SUN C.K. Chemical composition and inhibitory parameters of essential oil and extracts of Nandina domestica Thunb. to control foodborne pathogenic and spoilage bacteria. International Journal of Food Microbiology, 125, 2, 117-22, 2008.

3. BARBOSA-FILHO JM, VASCONCELOS THC, ALENCAR AA, BATISTA LM, OLIVEIRA RAG, GUEDES DN, FALCÃO HS, MOURA MD, DINIZ MFFM, MODESTO-FILHO J. Plants and their active constituents from South, Central, and North America with hypoglycemic activity. Revista Brasileira de Farmacognosia, 15, 392-413, 2005.

4. CASSEL, E.; VARGAS, R.M.F. Experiments and modeling of the Cymbopogon winterianus essential oil extraction by steam distillation. Journal of the Mexican Chemical Society, 50, 126-129, 2006.

5. DUARTE, M. C. T.; FigueiRA, G. M.; PEREIRA, B.; MAgAlhãES, P. M.; DELARMelinA, C. Atividade antimicrobina de extratos hidroalcoólicos de espécies da coleção de plantas medicinais CPQBA/UNICAMP. Revista Brasileira de Farmacognosia, 14, 6-8, 2004.

6. FERRONATT, R.; MARCHESAN, E. D, PEZENTI, P.; BEDNARSKI, F.; ONOFRE, S. B. Atividade antimicrobiana de óleos essenciais produzidos por Baccharis dracunculifolia D.C. e Baccharis uncinella D.C.(Asteraceae). Brazilian Journal of Pharmacognosy, 17, 224-230, 2007.

7. GBENOU, J. D.; AHOUNOU, J. F.; AKAKPO, H. B.; LALEYE, A.; ELÉONORE, Y.; GBAGUUIDI, F.; BABA-MOUSSA, L.; DARBOUX, R.; DANSOU, P.; MOUDACHIROU, M.; KOTCHONI, S.O. Phytochemical composition of Cymbopogon citratus and Eucalytus citriodora essential oils and their anti-inflammatory and analgesic properties on Wistar rats. Molecular biology reports, 20, 1127-34, 2013.

8. GIULlietTI, A. M.; HARLEY, R. M.; QUEIROZ, L. P.; WANDERLEY, M. G. L.; DEN BERG, C. V. Biodiversidade e conservação das plantas no Brasil. Revista Megadiversidade, 1, 52-61, 2005.

9. LOAYZA, I.; ABUJDER, D.; ARANDA, R.; JAKUPOVIC, J.; COLLIN, G.; DESLAURIERS, HE; JEAN, FI. 
Essential oils of Baccharis salicifolia, Baccharis latifolia and Baccharis dracunculifolia. Phytochemistry 38, 381-389, 1995.

10. LOAYZA, I.; COLLIN, G.; GAGNON, M.; DESLAURIERS, H.; DELLACASSA. Huiles essentielles de Baccharis latifolia, B. salicifolia de Bolivia de B. dracunculifolia em provenance d'Uruguay". Rivista Ital EPPOS Ed.Especial: 728-735, 1993.

11. LOPES, N. P.; KATO, M. J.; ANDRADE, E. H. A.; MAIA, J. G. S.; YOSHIDA, M.; Circadian and seasonal variation in the essential oil from Virola surinamensis leaves. Phytochemistry, 46, 689, 1997.

12. MATASYOH, J. C.; WAGARA I. N.; NAKAVUMA J. L.; KIBURAI, A. M. Chemical composition of Cymbopogon citrates essential oil and its effect on mycotoxigenic Aspergillus species. African Journal of Food Science. 5, 138-142, 2011.

13. OLIVEIRA, M.M.M.; BRUGNERA, D.F.; CARDOSO, M.G.; GUIMARÃES, L.G.L.; PICCOLI, R.H. Rendimento, composição química e atividade antilisterial de óleos essenciais de espécies de Cymbopogon. Revista Brasileira de Plantas Medicinais 13, 8-16, 2011.

14. PALÁ-PAÚl, J.; PÉREZ-ALONSO, M. J.; VELASCO-NEGUERUELA, A.; PALÁ-PAÚL, R.; SANZ, J.; CONEJERO, F. Plants as source of drugs. Biochemical Systematics and Ecology. 29: 663-665, 2001.

15. PITAREVIC, I.; KUFTINEC, J.; BLAEVIC, N.; KUŠTRAK, D. Seasonal variation of essential oil yield and composition of dalmatian sage, Salvia officinalis. Journal of Natural Products, 47, 3, 409$412,1984$.

16. SCHANEBERG, B.T., \& KHAN, I.A. Comparison of extraction methods for marker compounds in the essential oil of lemon grass By GC. Journal of Agricultural and Food Chemistry, 50, 13451349, 2002.

17. SCHWOB, I.; BESSIERE, J. M.; MASOTTI, V.; VIANO, J.; BIOCHEM. Syst. Ecol. 32, 735. 2004.

18. SILVA-SANTOS, A.; ANTUNES, A. M. S.; BIZZO, H. R.; D’AVILA, L. A. Análise Técnica, Econômica e de Tendências da Indústria Brasileira de Óleos Essenciais. Revista Brasileira de Plantas Medicinais, Papel Virtual, 8, 14, 2006.

19. SIMÕES, O. M. C.; SCHENKEL, R. P.; GOSMANN, G.; MELLO, P. C. J.; MENTZ, A. L.; PETROVICK, P. R. Farmacognosia da planta medicamento. Editora da Universidade Federal do Rio Grande do Sul. 1999.

20. VIANA, M. J. G. ; PALHANO, J. G. da; SANTOS, A. S. ; MAIA, J. G. S. ; ZOGHBI, M. G. B. ; ANDRADE, E. H. A. Visualização de acesso às informações sobre as plantas aromáticas da Amazônia; Construção de banco de dados de espécies investigadas quanto a composição química de seus óleos essenciais. Anais da Associação Brasileira de química. 47, 1, 57-63, 1998.

21. WENQIANG, G.; SHUFEN, L.; RUIXIANG, Y.; SHAOKUN, T.; CAN, Q. Comparison of essential oils of clove buds extracted with supercritical carbon dioxide and other three traditional extraction methods. Food Chemistry, 101, 1558-1564, 2007.

22. ZOGHBI,M. G. B. ; ANDRADE, E. H. A.; SANTOS, A. S.; SILVA, M. H. L. ; MAIA, J. G. S. Volatile constituents of the resins from protium subserratum (Engl.) Engl. And Tetragastris Panamensis (Engl.) Kuntz. Journal of Essential Oil Research, v. 10, p. 325-326, 1998. 